\title{
CARDINAL PRESERVING ELEMENTARY EMBEDDINGS
}

\author{
ANDRÉS EDUARDO CAICEDO
}

\begin{abstract}
Say that an elementary embedding $j: N \rightarrow M$ is cardinal preserving if $\mathrm{CAR}^{M}=$ $\mathrm{CAR}^{N}=\mathrm{CAR}$. We show that if PFA holds then there are no cardinal preserving elementary embeddings $j: M \rightarrow V$. We also show that no ultrapower embedding $j: V \rightarrow M$ induced by a set extender is cardinal preserving, and present some results on the large cardinal strength of the assumption that there is a cardinal preserving $j: V \rightarrow M$.
\end{abstract}

§1. Introduction. This paper is the first of a series attempting to investigate the structure of (not necessarily fine structural) inner models of the set theoretic universe under assumptions of two kinds:

1. Forcing axioms, holding either in the universe $V$ of all sets or in both $V$ and the inner model under study, and

2. Agreement between (some of) the cardinals of $V$ and the cardinals of the inner model.

I try to be as self-contained as is reasonably possible, given the technical nature of the problems under consideration. The notation is standard, as in Jech [8]. I assume familiarity with inner model theory; for fine structural background and notation, the reader is urged to consult Steel [19] and Mitchell [15].

In the remainder of this introduction, I include some general observations on large cardinal theory, forcing axioms, and fine structure, and state the main results of the paper.

Consider set theory with the axiom of choice as formalized by the GödelBernays axioms GBC, so we can freely treat proper classes. An inner model (or simply, a model) is a transitive class model $M$ of the Zermelo-Fraenkel ZFC axioms containing all the ordinals. If $M$ is a model and $\varphi$ is a statement, $\varphi^{M}$ is the assertion that $\varphi$ holds in $M$. If $\tau$ is a definable term, $\tau^{M}$ indicates the interpretation of $\tau$ inside $M$. Denote by ORD the class of ordinals and by CAR the class of cardinals. The cofinality of an ordinal $\alpha$ is denoted $\operatorname{cf}(\alpha)$. All our embeddings are elementary and non-trivial, and the classes involved are inner models; the critical point of such an embedding $j$ is denoted $\mathrm{cp}(j)$.

Logic Colloquium '07

Edited by Françoise Delon, Ulrich Kohlenbach, Penelope Maddy, and Frank Stephan

Lecture Notes in Logic, 35

(c) 2009, Association for Symbolic Logic 
1.1. Large cardinal axioms. See Kanamori [10] as a general reference for large cardinals. It is well-known that the GBC axioms fall quite short of providing a complete picture of the universe of sets. Among the many independent extensions that have been studied, two sets of statements have been isolated as natural candidates to add to the basic axioms: large cardinal axioms and forcing axioms. By far, large cardinal axioms are better understood and more readily accepted. There is no formal definition of what a large cardinal is, but a few features can be distinguished. Typically, they are regular cardinals $\kappa$ such that $V_{\kappa}$ is itself a model of ZFC and, more importantly for present purposes, one can associate to $\kappa$ a family of elementary embeddings $j: V \rightarrow M$ where $M$ is a transitive class. The association is usually (as in the case of measurable, strong or supercompact cardinals) that $\kappa$ is the critical point $\operatorname{cp}(j)$ of $j$, the first ordinal $\alpha$ such that $j(\alpha)>\alpha$, but it can take other shapes, as is the case with Woodin cardinals.

An important remark is that these notions can be stated in terms of the existence of certain ultrafilters or systems of ultrafilters called extenders, the connection being given by an analogue of the model theoretic ultrapower construction. An extender is essentially (a family of ultrafilters coding together) a fragment of an elementary embedding, and it is by now a standard device; good expositions and definitions can be found in Jech [8, Chapter 20], Kanamori $[10, \S 26]$, and Steel $[19, \S 2.1]$, among others. Briefly:

For a set $X$ and a cardinal $\kappa$, let

$$
[X]^{\kappa}=\{Y \subseteq X:|Y|=\kappa\}
$$

and define $[X]^{<\kappa}$ and $[X]^{\leq \kappa}$ similarly.

Definition 1.1. Let $\kappa$ be a cardinal and let $\lambda>\kappa$. A non-trivial $(\kappa, \lambda)$ extender $E$ is a sequence $\left(E_{a}: a \in[\lambda]^{<\omega}\right)$ such that there is some $\zeta \geq \kappa$ for which the following hold:

1. For each $a \in[\lambda]^{<\omega}, E_{a}$ is a $\kappa$-complete ultrafilter over $[\zeta]^{|a|}$. We call $\kappa=\operatorname{cp}(E)$ the critical point of $E$ and $\lambda=\operatorname{lh}(E)$ the length of $E$.

2. (Non-triviality) For at least one $a \in[\lambda]^{<\omega}, E_{a}$ is not $\kappa^{+}$-complete.

3. For each $\xi \in \zeta$ there is $a \in[\lambda]^{<\omega}$ such that $\left\{s \in[\zeta]^{|a|}: \xi \in s\right\} \in E_{a}$.

4. (Coherence or Compatibility) Whenever $a \subseteq b \in[\lambda]^{<\omega}$ let $\pi_{b a}:[\zeta]^{|b|} \rightarrow$ $[\zeta]^{|a|}$ be the projection map given by

$$
\pi_{b a}(s)=\pi_{s}[a]
$$

where $\pi_{s}:(b,<) \rightarrow(s,<)$ is the unique order isomorphism. Then, for $a \subseteq b \in[\lambda]^{<\omega}$ and $X \subseteq[\zeta]^{|a|}$,

$$
X \in E_{a} \text { iff }\left\{s: \pi_{b a}(s) \in X\right\} \in E_{b} .
$$

5. (Normality) If $a \in[\lambda]^{<\omega}, i<|a|$, and $f:[\zeta]^{|a|} \rightarrow V$ is such that

$$
\left\{s \in[\zeta]^{|a|}: f(s)<s_{i}\right\} \in E_{a}
$$


(where $s_{i}$ is the $i$-th element of $s$ in increasing order) then there is $b \in[\lambda]^{<\omega}$ with $a \subseteq b$ and $k<|b|$ such that

$$
\left\{s \in[\zeta]^{|b|}: f\left(\pi_{b a}(s)\right)=s_{k}\right\} \in E_{b},
$$

where $\pi_{b a}$ is as above.

The relation between extenders and elementary embeddings is described in the following result:

Lemma 1.2. 1. Assume $j: V \rightarrow N$ is elementary. Let $\kappa=\operatorname{cp}(j)$ and $\lambda>\kappa$. Let $\zeta$ be (least) such that $j(\zeta) \geq \lambda$. For all $a \in[\lambda]^{<\omega}$, define

$$
E_{a}:=\left\{X \subseteq[\zeta]^{|a|}: a \in j(X)\right\} .
$$

Then $E=\left(E_{a}: a \in[\lambda]^{<\omega}\right)$ is a $(\kappa, \lambda)$-extender.

2. Conversely, given a $(\kappa, \lambda)$-extender $E$ with each $E_{a}$ over $[\zeta]^{|a|}$, there is an elementary embedding $j: V \rightarrow N$ such that

(a) $\kappa=\operatorname{cp}(j)$, $\zeta$ is least such that $\lambda \leq j(\zeta)$, and for all $a \in[\lambda]^{<\omega}$, $E_{a}=\left\{X \subseteq[\zeta]^{|a|}: a \in j(X)\right\}$. Moreover,

(b) $N=\left\{j(f)(s): s \in[\lambda]^{<\omega}\right.$ and $\left.f:[\zeta]^{|s|} \rightarrow V\right\}$.

For a proof see any of the references cited above; the model $N$ and the embedding $j$ in item 2 are obtained as the direct limit of the system that consists of the ultrapowers ult $\left(V, E_{a}\right)$ and their associated embeddings; that this system is directed follows from the compatibility condition in Definition 1.1. It is customary to refer to this direct limit (or its transitive collapse) as ult $(V, E)$. Our interest in Lemma 1.2 lies in the following corollary, an immediate consequence of Lemma 1.2 2(b):

CoRollary 1.3. Let $E$ be a $(\kappa, \lambda)$-extender with each $E_{a}$ over $[\zeta]^{|a|}$, and let $j_{E}: V \rightarrow N$ be the associated embedding. Then, for any $\xi, j_{E}(\xi)<$ $\left(\left|\xi^{\zeta}\right| \cdot|\lambda|\right)^{+}$.

We have mentioned examples of large cardinals. However, what is accepted as a large cardinal axiom is more general. For example, the existence of certain mice is a large cardinal axiom. They do not imply that large cardinals exist in $V$, but rather in certain inner models.

A compelling reason for accepting large cardinal axioms comes from the heuristic realization that natural statements that do not involve large cardinals in their formulation can be shown equiconsistent with certain large cardinal axioms. At the moment this is more a conjecture than a fact (hence the informal adjective "natural" in the description just given, intended to exclude, for example, pathological statements such as those generated by means of Gödel sentences), but it is a widely accepted state of affairs having been verified for a varied class of examples; to name just a few: reflection of stationary sets, determinacy hypotheses, or the failure of the singular cardinals hypothesis 
$\mathrm{SCH}$. Recall that $\mathrm{SCH}$ is the statement that for all cardinals $\kappa$,

$$
\kappa^{\operatorname{cf}(\kappa)}=2^{\mathrm{cf}(\kappa)}+\kappa^{+}
$$

it follows from work of Woodin and Gitik (see Jech [8, Chapter 36] and references therein) that the failure of $\mathrm{SCH}$ is equiconsistent with the existence of a cardinal $\kappa$ of Mitchell order $o(\kappa)=\kappa^{++}$. Baumgartner showed that the proper forcing axiom PFA (see subsection 1.2) is consistent relative to the existence of supercompact cardinals, see Shelah [18, Chapter VII]; it is widely expected that, once fine structural inner model theory has been developed enough, it will be shown that PFA is in fact equiconsistent with a strong large cardinal axiom. The best available result to date is from Jensen-SchimmerlingSchindler-Steel [9], where it is shown that PFA implies the existence of nondomestic mice (see subsection 1.3), see also Andretta-Neeman-Steel [1].

1.2. Forcing axioms. As said before, forcing axioms are not yet so widely understood. While some set theorists go as far as considering them natural statements, what seems to be the consensus is that they formalize a very desirable feature of the universe of sets. Namely, one would like the universe to be as "wide" or "saturated" as possible. The way in which forcing axioms formalize this desire is by stating that certain sets that can be added by certain forcing posets already exist. There are restrictions on the class of posets to be considered and on how generic these sets that would have been added can actually be. By loosening or increasing the restrictions, a variety of forcing axioms can be identified. The most widely used and best known is by far Martin's axiom MA, see Martin-Solovay [14], but this is not a strong enough statement to even decide the size of the continuum. Strong forcing axioms are much stronger than MA both in consequences and in consistency strength. Typical examples are the proper forcing axiom PFA introduced by Baumgartner and Shelah, see Baumgartner [2] and Shelah [18, Chapter VII], and Martin's maximum MM introduced in the groundbreaking paper Foreman-Magidor-Shelah [5].

For $\Gamma$ a collection of forcing notions, let $F A(\Gamma)$ be the statement that for any $\mathbb{P} \in \Gamma$ and any $\omega_{1}$-many given dense subsets of $\mathbb{P}$ there is (in $V$ ) a filter $G \subseteq \mathbb{P}$ sufficiently generic in the sense that $G$ meets each of these dense filters.

Assume that $X$ is an uncountable set. A set $S \subseteq[X]^{\omega}$ is said to be stationary if for any function $f:[X]^{<\omega} \rightarrow X$ there is a set $y \in S$ closed under $f$. A forcing $\mathbb{P}$ is proper iff for any stationary set $S$, it is still the case in $V^{\mathbb{P}}$ that $S$ is stationary. In particular, uncountable sets in $V$ are still uncountable in $V^{\mathbb{P}}$ and thus $\omega_{1}^{V}=\omega_{1}^{V^{\mathbb{P}}}$. The proper forcing axiom PFA is the statement FA(Proper), where Proper is the class of proper posets.

Given a poset $\mathbb{P}$, it can be shown, see Foreman-Magidor-Shelah [5], that $\mathrm{FA}(\{\mathbb{P}\})$ fails if there is a stationary subset $S$ of $\omega_{1}$ that is no longer stationary in $V^{\mathbb{P}}$ (one says that the stationarity of $S$ was not preserved). Martin's maximum MM is the statement that $\mathrm{FA}(\Gamma)$ holds, where $\Gamma$ is the class of posets 
that preserve stationary subsets of $\omega_{1}$. Obviously, $\mathrm{MM}$ is the strongest forcing axiom possible, and in particular it implies PFA. As with PFA, it is consistent relative to the existence of a supercompact cardinal.

Many consequences of PFA can be considered natural features of the universe of sets (for example, the failure of the continuum hypothesis, the failure of square principles, the singular cardinal hypothesis, determinacy in $L(\mathbb{R})$, and generic absoluteness of $L(\mathbb{R})$; see for example Bekkali [3], Todorčević [20], Viale [22], and Jensen-Schimmerling-Schindler-Steel [9]) thus providing evidence for its acceptance as a natural extension of ZFC. However, even if one does not consider PFA to be "natural", it seems reasonable that some common features of forcing axioms and other similar strong reflection principles will eventually be considered as natural as large cardinal axioms.

Recall:

Definition 1.4. Let $\kappa$ be an uncountable cardinal.

1. $\kappa$ is strongly compact iff for any set $S$, any $\kappa$-complete filter over $S$ can be extended to a $\kappa$-complete ultrafilter over $S$.

2. Given a set $X$, a filter $F$ over $[X]^{<\kappa}$ is fine iff it is $\kappa$-complete and, for all $x \in X$,

$$
\left\{\sigma \in[X]^{<\kappa}: x \in \sigma\right\} \in F .
$$

3. Given a cardinal $\lambda \geq \kappa$, say that $\kappa$ is $\lambda$-strongly compact or, simply, $\lambda$-compact iff there is a fine ultrafilter over $[\lambda]^{<\kappa}$.

The following characterization of strong compactness in terms of elementary embeddings will be useful in Section 3, see Kanamori [10, Theorem 22.17]:

THEOREM 1.5. Let $\omega<\kappa \leq \lambda$. Then the following are equivalent:

1. $\kappa$ is $\lambda$-compact.

2. There is an elementary $j: V \rightarrow N$ with $\mathrm{cp}(j)=\kappa$ and such that for any $X \subseteq N$ with $|X| \leq \lambda$, there is $Y \in N$ with $X \subseteq Y$ and $N \models|Y|<j(\kappa)$.

3. For any set $S$, any $\kappa$-complete filter over $S$ generated by at most $\lambda$ sets can be extended to a $\kappa$-complete ultrafilter over $S$.

We have occasion to use some covering properties introduced in the highly recommended Viale [23]. We proceed to recall the relevant notions and results:

Definition 1.6 (Viale). Let $\lambda<\kappa$ be regular cardinals. A collection of sets $\mathcal{D}=(K(\alpha, \beta): \alpha<\lambda, \beta<\kappa)$ is a $\lambda$-covering matrix for $\kappa$ iff the following conditions are met:

1. $\beta \subseteq \bigcup_{\alpha<\lambda} K(\alpha, \beta)$ for all $\beta<\kappa$.

2. $K(\alpha, \beta) \subsetneq K(\eta, \beta)$ for all $\beta<\kappa$ and all $\alpha<\eta<\lambda$.

3. For all $\alpha<\lambda$ and all $\gamma<\beta<\kappa$ there is $\eta<\lambda$ such that $K(\alpha, \gamma) \subseteq$ $K(\eta, \beta)$.

4. For all $X \in[\kappa]^{\leq \lambda}$ there is $\gamma_{X}<\kappa$ such that for all $\beta<\kappa$ and $\eta<\lambda$ there is $\alpha<\lambda$ such that $K(\eta, \beta) \cap X \subseteq K\left(\alpha, \gamma_{X}\right)$. 
Definition 1.7 (Viale). If $\kappa>\lambda$ are regular cardinals, the covering property $\mathrm{CP}(\kappa, \lambda)$ holds iff for every $\lambda$-covering matrix $\mathcal{D}$ for $\kappa$ there is an $A \in[\kappa]^{\kappa}$ such that

$$
[A]^{\lambda} \subseteq \bigcup\left\{[K(\alpha, \beta)]^{\lambda}: \alpha<\lambda, \beta<\kappa\right\} .
$$

The definition of the combinatorial principle $\square(\kappa)$ mentioned below can be found, for example, in Moore [16].

THEOREM 1.8 (Viale). 1. CP $(\kappa, \omega)$ implies that $\square(\kappa)$ fails.

2. If $\kappa$ is singular, $\mathrm{cf}(\kappa)=\lambda, 2^{\lambda}<\kappa$, and $\mathrm{CP}\left(\kappa^{+}, \lambda\right)$ holds, then $\kappa^{\lambda}=\kappa^{+}$.

3. $\mathrm{CP}\left(\kappa^{+}, \omega\right)$ for all singular $\kappa$ of cofinality $\omega$ implies the singular cardinal hypothesis $\mathrm{SCH}$.

4. PFA implies $\mathrm{CP}\left(\kappa^{+}, \omega\right)$ for all singular $\kappa$ of cofinality $\omega$.

5. If $\lambda$ is strongly compact, then $\mathrm{CP}(\kappa, \theta)$ holds for all regular $\theta<\lambda$ and all regular $\kappa \geq \lambda$.

6. If $\mathrm{CP}\left(\kappa^{+}, \theta\right)$ holds and $M$ is an inner model such that $\kappa$ is regular in $M$ and $\left(\kappa^{+}\right)^{M}=\kappa^{+}$, then $\operatorname{cf}(\kappa) \neq \theta$.

7. If PFA holds and $M$ is an inner model with the same cardinals, then $M$ computes correctly all ordinals of cofinality $\omega$.

1.3. Fine structure. See Steel [19], Mitchell [15], and references within for historical references and details on the notions and results mentioned here. What follows owes much to expositions by Ketchersid. Inner model theory is a rather technical area in set theory, and by necessity the presentation I make of it here is somewhat of a caricature. Gödel defined the constructible universe $L$; it is not really a good model of set theory as currently understood since it does not contain any significant large cardinals. On the other hand, its theory admits a very detailed analysis (known as fine structure theory and introduced by Jensen) by studying how new sets are added in the inductive construction of $L$ in terms of the complexity of their definitions. One can generalize $L$ to models of the form $L[\mathcal{E}]$, the constructible universe built from an additional predicate $\mathcal{E}$, and by allowing $\mathcal{E}$ to code (one or several) elementary embeddings, $L[\mathcal{E}]$ can be made to model a substantial fragment of the large cardinal hierarchy. However, to replicate the fine structural analysis of $L$ in this larger generality requires substantially new ideas, since the predicate $\mathcal{E}$ should be chosen very carefully in order to obtain some kind of canonicity.

The (partial) solution devised by Mitchell and Steel, following a long line of research including work of Jensen, Solovay, Dodd and Mitchell, among others, is to let $\mathcal{E}=\left\langle E_{\alpha}: \alpha<\beta\right\rangle$ be a coherent sequence of extenders. By reference to just a extender $E$, i.e., without need of knowing the domain of the embedding being coded, one can recover the critical point of this embedding, which justifies talking of $\mathrm{cp}(E)$. Also, as long as some agreement conditions are satisfied, an ultrapower ult $(M, E)$ of a model $M$ by an extender $E$ can be formed, with critical point $\mathrm{cp}(E)$, even if $M$ was not the domain of the original 
embedding coded by $E$ or if $E \notin M$. Coherency is a technical requirement; each $E_{\alpha}$ is either $\emptyset$ or else it is an extender over $L_{\alpha}[\mathcal{E}\lceil\alpha]$; moreover, if $j: L_{\alpha}\left[\mathcal{E}\lceil\alpha] \rightarrow \operatorname{ult}\left(L_{\alpha}\left[\mathcal{E}\lceil\alpha], E_{\alpha}\right)\right.\right.$ is the ultrapower embedding by $E_{\alpha}$, then $j(\mathcal{E})\left\lceil\alpha=\mathcal{E}\left\lceil\alpha\right.\right.$ and $j(\mathcal{E})_{\alpha}=\emptyset$. (In this description I have ignored a few details, in particular, some technical remarks related to the well-foundedness of the target model.)

A potential premouse $(\mathrm{ppm})$ is a structure $\mathcal{M}=\left\langle L_{\alpha}\left[\mathcal{E}\lceil\alpha], E_{\alpha}\right\rangle\right.$ where $\mathcal{E}$ is a coherent sequence of extenders. These are structures that "look like" initial segments of one of the canonical models one wants to build. Part of what the canonicity of the construction requires is an analogue of some of the nice condensation properties of $L$. This is called the initial segment condition, and a ppm satisfying this is called a premouse.

For $\mathcal{M}$ as above and $\beta \leq \alpha$, let $\mathcal{M} \mid \beta=L_{\beta}\left[\mathcal{E}\lceil\beta]\right.$ and $\mathcal{M} \| \beta=\left\langle L_{\beta}\left[\mathcal{E}\lceil\beta], E_{\beta}\right\rangle\right.$. A key issue when studying premice is the question of iterability. Two premice $\mathcal{M}$ and $\mathcal{N}$ are lined up if one is an initial segment of the other, i.e., if for some $\beta, \mathcal{N}=\mathcal{M} \| \beta$ or $\mathcal{M}=\mathcal{N} \| \beta$. To compare two premice $\mathcal{M}$ and $\mathcal{N}$ means to produce from them two (other) lined up premice $\mathcal{M}^{*}$ and $\mathcal{N}^{*}$. To do this, the notion of iteration tree, due to Martin and Steel, is required. In short, nice inner models for small large cardinals can be compared by iterating ultrapowers, see Jech [8, Chapter 19]: One looks at the first ordinal $\beta$ where $\mathcal{M}\|\beta \neq \mathcal{N}\| \beta$. This disagreement must come from their top measures being different, $E_{\beta}^{\mathcal{M}} \neq E_{\beta}^{\mathcal{N}}$. Forming $\mathcal{M}^{\prime}=\operatorname{ult}\left(\mathcal{M} \mid \beta, E_{\beta}^{\mathcal{M}}\right)$ and $\mathcal{N}^{\prime}=\operatorname{ult}\left(\mathcal{N} \mid \beta, E_{\beta}^{\mathcal{N}}\right)$, the coherency property of the extender sequences implies that $\mathcal{M}^{\prime}\left\|\beta=\mathcal{N}^{\prime}\right\| \beta$, i.e., we have effectively removed a disagreement. The process is continued (taking direct limits at limit stages) until (if) lined up models are produced. It is a remarkable result of Kunen that this is indeed the case for models for one measurable cardinal, and this result can be generalized. The iterations so obtained are essentially linear iterations, and this linearity seriously bounds both the complexity of the reals that can belong to such inner models and their large cardinals. Martin and Steel found a non-linear method of iterating ultrapowers of inner models in the region of Woodin cardinals. These models give then rise to iteration trees, trees of structures with embeddings between the models appearing along their branches. If two models are compared this way, at limit stages of the comparison process, different possibilities on how to continue the trees may arise, and the existence of these choices increases the complexity of their comparison process and explains why these models allow more complicated reals and large cardinals than those appearing in linearly iterable models. From the existence of enough large cardinals one can deduce the existence of nice models $M$, i.e., iterable (in an appropriate sense that allows us to carry out the comparisons mentioned above) models of enough set theory with roughly the same large cardinals. Exactly how far in the large cardinal hierarchy this process 
can go is open, but models with many Woodin cardinals can be obtained this way.

A premouse $\mathcal{M}$ is collapsing if every element of $\mathcal{M}$ is $\Sigma_{1}$-definable over $\mathcal{M}$ from no parameters - in particular, $\mathcal{M}$ is countable. Collapsing (sufficiently iterable) premice are already lined up. The most famous example of a collapsing mouse (and the smallest one) is zero sharp, $0^{\sharp}$, this is an iterable model of the form $0^{\sharp}=M_{0}^{\sharp}=\left\langle L_{\alpha}, E_{\alpha}\right\rangle$ where $0^{\sharp} \models " E_{\alpha}$ is a measure on $\operatorname{cp}\left(E_{\alpha}\right)$ ", with $\alpha$ chosen as least as possible. Hence, $0^{\sharp}$ resembles an inner model with a measurable cardinal, except that by considering the next level of the constructible hierarchy, the universe is collapsed to $\omega$. Other examples include $0^{\dagger}$, which is a model with a genuine measure and a top extender, and $M_{l}^{\sharp}$, $l \leq \omega$, models with $l$ Woodin cardinals and a top extender. Since these models are lined up, one can talk of a mouse being "below a Woodin cardinal", for example. Work of Steel, Martin, Woodin and Neeman has shown that $\Sigma_{\sim n+1}^{1}$-determinacy is equivalent to the existence and iterability of $M_{n}^{\sharp}(x)$ for all reals $x$, where these models are defined as $M_{n}^{\sharp}$, but the real $x$ is added as an element at the bottom stage (for $n=0$ this is a result of Martin and Harrington). Similarly, $A D^{L(\mathbb{R})}$ in all set generic extensions is equivalent to the existence and iterability of $M_{\omega}^{\sharp}$ and this is in turn equivalent to the generic absoluteness of $L(\mathbb{R})$. Below, we mention non-domestic premice; these are premice $\mathcal{M}$ such that there is an ordinal $v \leq \mathrm{ORD}^{\mathcal{M}}$ such that in $\mathcal{M} \| v$, $\kappa=\operatorname{cp}\left(E_{v}\right)$ is a limit of Woodin cardinals and of cardinals strong up to $\kappa$, see Andretta-Neeman-Steel [1].

The model $K$, the core model, is an iterable model built from a coherent sequence of extenders as above. It is intended to faithfully represent the large cardinal structure of the universe. Its existence is in general an open problem (and requires some additional technical assumptions to ensure its iterability), but it has been successfully identified as long as this large cardinal structure is not too complicated. For example, it is just $L$ if $0^{\sharp}$ does not exist. Much like $L, K$ is quite canonical; for example, it is definable, and its definition is invariant under forcing. It satisfies the weak covering property, namely that $\left(\lambda^{+}\right)^{K}=\lambda^{+}$for all $V$-singular cardinals $\lambda$; when $K=L$ (which is to say, when $0^{\sharp}$ does not exist) this is a consequence of Jensen's celebrated covering lemma. An important technical feature of $K$ is its rigidity. This means that there are no elementary embeddings $j: K \rightarrow K$. In fact, a standard technique to show that an assumption $\varphi$ implies the existence of mice capturing certain large cardinals, consists on attempting to build $K$ under the assumption that $\varphi$ holds and the mice in question do not exist, and then proceeding to show that $K$ is non-rigid.

1.4. Results. We study embeddings $j: V \rightarrow M$ or $j: M \rightarrow V$ where the model $M$ computes cardinals correctly, i.e., $\mathrm{CAR}^{M}=\mathrm{CAR}$. We call these 
embeddings cardinal preserving. The expectation is that they do not exist, and the results of this paper can be seen as a step towards confirming this expectation. In Section 2 we show:

THEOREM 1.9. If PFA holds, then there are no cardinal preserving embeddings $j: M \rightarrow V$.

Theorem 1.9 follows Viale's Theorem 1.8 via a structural restriction we identify, see Theorem 2.5.

In Section 3 we study cardinal preserving embeddings $j: V \rightarrow M$, and prove:

THEOREM 1.10. If $E$ is a set extender and $j: V \rightarrow M$ is the corresponding ultrapower embedding, then $j$ is not cardinal preserving.

Theorem 1.10 also makes use of (the proof of) Viale's Theorem 1.8. Next, we show that cardinal preserving embeddings $j: V \rightarrow M$ have significant large cardinal strength, both locally (e.g., $\operatorname{cp}(j)$ is $\mathrm{cp}(j)^{+}$-compact and much more) and globally (e.g., $V$ is closed under sharps, and significantly more).

All the lower bounds in consistency strength obtained in this paper, Theorems 2.11, 3.2 and 3.3, except for the combinatorial Theorem 3.7 (joint with H. Woodin), follow from fine structural considerations that trace back to violations of the appropriate covering lemma, see Mitchell [15]; Steel [19] and Mitchell [15] provide all unexplained notation and background results used in these proofs.

\section{$\S 2$. Embeddings into $V$.}

Question 2.1. Assume $j: M \rightarrow N$ is an elementary embedding. Can we have $\mathrm{CAR}^{M}=\mathrm{CAR}^{N}=\mathrm{CAR}$ ?

We suspect the answer is no. In this paper we take the first step towards this question by considering the case where either $M$ or $N$ is $V$, and analyzing the structure of such cardinal preserving elementary embeddings.

Notice that such embeddings seem rather difficult to attain by standard means. For example:

THEOREM 2.2 (Hamkins [6]). No embedding $j: V \rightarrow V[G]$ can be produced by set forcing.

Embeddings into $V$ can be produced by class forcing, see below, or by means of indiscernibles, see Vickers-Welch [24].

Also, if $E$ is a set extender and $j: V \rightarrow M$ is the corresponding ultrapower embedding, then $j$ is not cardinal preserving. We present here a quick argument under the additional assumption of the singular cardinal hypothesis

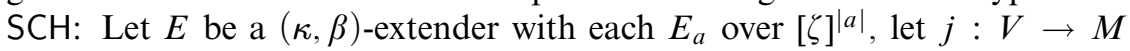
be the corresponding embedding, and let $\lambda$ be a sufficiently large strong limit 
cardinal of cofinality $\kappa$. Then $\lambda<\lambda^{+}=\lambda^{|\xi|}$ by $\mathrm{SCH}$, and $\lambda<j(\lambda)$, since $\operatorname{cf}(\lambda)=\kappa$. But

$$
\lambda^{|\xi|} \leq j\left(\lambda^{|\xi|}\right)<\left(\lambda^{|\xi|}|\beta|\right)^{+}
$$

by Corollary 1.3. So, if $j$ is cardinal preserving, then $j\left(\lambda^{|\xi|}\right)=\lambda^{|\xi|}$, and $\lambda<j(\lambda)<j\left(\lambda^{|\xi|}\right)=\lambda^{+}$, contradiction. We present a proof without the assumption of $\mathrm{SCH}$ in Theorem 3.6.

Initial impulse for this line of research came from the following conjecture:

Conjecture 2.3. Assume PFA. Let $M$ be an inner model that computes cardinals correctly and contains all the reals. Then $\mathrm{ORD}^{\omega} \subset M$.

This is not the place to explain why we expect Conjecture 2.3 to be the case; see Viale [22, 23] and Caicedo-Veličović [4]. As a quick motivation, recall that Moore has shown that MRP, a consequence of PFA (see Moore [16]), fails after adding a Prikry sequence, and Viale [21] has generalized this result.

We can help clarify the role of the assumption of agreement of cardinals:

1. Suppose for example that $M$ has enough large cardinals. Then there are forcing extensions $V$ of $M$ where PFA holds, but $V$ and $M$ have little else in common so, clearly, additional agreement between $V$ and $M$ is required.

2. Or suppose that $\kappa$ is measurable. Let $M$ be the result of iterating $\omega$-many times a given measure on $\kappa$ and let $j: V \rightarrow M$ be the corresponding embedding. Then $\mathrm{cf}(j(\kappa))=\omega, 2^{\kappa}<j(\kappa)<\left(2^{\kappa}\right)^{+}$and, in $M, j(\kappa)$ is inaccessible (by elementarity of $j$ ). It follows that $M$ is not closed under $\omega$-sequences and $\mathbb{R} \subset M$. Of course, we can also assume in this situation that PFA holds in $V$.

3. Also, if $M$ has a proper class of completely Jónsson cardinals, $\mathbb{P}_{\infty}$ is the class stationary tower (see Larson [13]), $G$ is $\mathbb{P}_{\infty}$-generic over $M$, and $V=M[G]$, then there is (definably in the structure $(V, M, G)$ ) an elementary

$$
j: M \rightarrow V,
$$

and we can arrange that $\mathrm{cp}(j)$ is arbitrarily high and $\mathrm{cf}^{V}(\mathrm{cp}(j))=\omega$. However, in the situation of this example, cardinals are always collapsed: The critical point of such a $j$ is in $M$ an uncountable regular cardinal $\lambda$. Let $a=S_{\omega}^{\lambda}$, the set of ordinals below $\lambda$ of cofinality $\omega$. By elementary properties of $\mathbb{P}_{\infty}, \operatorname{cp}(j)=\lambda$ and $\operatorname{cf}(\lambda)=\omega$ iff $a \in G$. For example, if $a \in G$ then $j[\cup a] \in j(a)$, so $j[\lambda]$ will be an ordinal of cofinality $\omega$ below $j(\lambda)$. Of course, this means that $j[\lambda]$ must equal $\lambda$, and $\lambda$ is therefore the critical point of $j$. If $\lambda$ is a successor cardinal in $M$, then it is collapsed in $V$ but if it is inaccessible in $M$, then it is preserved even though its cofinality changes. Decoding definitions, $\lambda^{+}$is preserved in $M[G]$ iff the intersection of $a$ with the set of subsets of $\lambda^{+}$of order type $\lambda^{+}$is stationary, and this intersection is in $G$. In particular, $\lambda^{+}$is a Jónsson 
cardinal in $M$, see Kanamori $[10, \S 8]$. On the other hand, no successor of a regular cardinal is Jónsson, so $\lambda^{+}$has to be collapsed.

The following result indicates that under PFA the existence of a cardinal preserving $j: M \rightarrow V$ would contradict Conjecture 2.3; this motivated Theorem 1.9 stating that no such embeddings can occur under PFA. Theorem 1.9 is proven in the following subsection; we show that if $j: M \rightarrow V$ is cardinal preserving, then $M$ computes incorrectly many cofinalities (which is significantly stronger than $\left.\mathrm{ORD}^{\omega} \not \subset M\right)$.

THEOREM 2.4 (Foreman, see Vickers-Welch [24]). If $j: M \rightarrow V$ is elementary, then $\mathrm{ORD}^{\omega} \not \subset M$.

Thus, one expects that Question 2.1 has a negative answer under PFA (or even provably in GBC).

2.1. Discontinuities. In the case of cardinal preserving embeddings $j$ : $M \rightarrow V$ a rather bizarre picture is known.

THeOREM 2.5. Suppose that $j: M \rightarrow V$ is cardinal preserving. Let $\kappa=$ $\operatorname{cp}(j)$. Then for all $\lambda>\kappa, j(\lambda)>\lambda$. In particular, if $j[\lambda] \subseteq \lambda$, then $\operatorname{cf}^{M}(\lambda) \geq \kappa$.

Proof. We proceed by contradiction, assuming $j: M \rightarrow V$ is a counterexample. Let $\kappa=\operatorname{cp}(j)$. We want to show that if $\lambda>\kappa$ then $j(\lambda)>\lambda$. Let $\lambda$ be the first counterexample. Then $\lambda$ is a cardinal: If $M=\tau=|\lambda|$, then $j(\tau)=|j(\lambda)|=|\lambda|$, since $j(\lambda)=\lambda$. Since $j(\tau)$ is a cardinal and in $M$ there is a bijection between $\lambda$ and $\tau, j(\tau)=|\lambda|=\tau$.

The argument now splits into cases, depending on whether $\lambda$ is singular or not.

CASE $1 . \lambda$ is singular.

The following notions and result are essential to pcf theory, see Shelah [17, Chapter II].

Definition 2.6. 1. Let $I$ be an ideal on a set $X$ and $f, g: X \rightarrow$ ORD. Then $f<_{I} g$ iff

$$
\{x \in X: g(x) \leq f(x)\} \in I .
$$

2. Given a cardinal $\tau$, let $\mathcal{J}_{\tau}^{b d}$ be the ideal of bounded subsets of $\tau$.

Definition 2.7. Let $\mu$ be singular. A scale for $\mu$ is a tuple $(\vec{\mu}, \vec{f})$ such that

1. $\vec{\mu}=\left(\mu_{i}: i<\operatorname{cf}(\mu)\right)$ is an increasing sequence of regular cardinals cofinal in $\mu$.

2. $\vec{f}=\left(f_{\alpha}: \alpha<\mu^{+}\right)$is a sequence of functions such that
(a) $f_{\alpha} \in \prod_{i<\mathrm{cf}(\mu)} \mu_{i}$ for all $\alpha<\mu^{+}$,
(b) If $\beta<\gamma<\mu^{+}$, then $f_{\beta}<_{\mathcal{J}_{\operatorname{cf}(\mu)}^{b d}} f_{\gamma}$, and
(c) If $f \in \prod_{i<\mathrm{cf}(\mu)} \mu_{i}$, then there is $\alpha<\mu^{+}$such that $f<_{\mathcal{J}_{\mathrm{cf}(\mu)}^{b d}} f_{\alpha}$. 
Theorem 2.8 (Shelah $[17$, Chapter II]). Let $\lambda$ be singular. Then there is a scale for $\lambda$.

Let $(\vec{\lambda}, \vec{f})$ be a scale for $\lambda$ in $M$. We may assume that $\lambda_{0}>\kappa$, so $j\left(\lambda_{i}\right)>\lambda_{i}$ for all $i<\mathrm{cf}^{M}(\lambda)$.

Let $\delta=\operatorname{cf}^{M}(\lambda)$. Then $j(\delta)=\operatorname{cf}(j(\lambda))=\operatorname{cf}(\lambda) \leq \operatorname{cf}^{M}(\lambda)=\delta$, so $\delta=j(\delta)$ and therefore, since $\lambda$ is the least fixed point of $j$ above $\kappa, \delta<\kappa$ and $j(\vec{\lambda})=$ $j[\vec{\lambda}]$.

Now we use an argument of Zapletal [25]. We have that $(j(\vec{\lambda}), j(\vec{f}))$ is a scale for $\lambda$ in $V$. Notice that $\lambda^{+}=j\left(\lambda^{+}\right)$, so $j[\vec{f}]=\left(j\left(f_{\alpha}\right): \alpha<\lambda^{+}\right)$is a scale for $\lambda$ as well.

Now consider $g \in \prod_{i<\delta} j\left(\lambda_{i}\right)$ given by

$$
g(i)=\sup \left(j[\lambda] \cap j\left(\lambda_{i}\right)\right)=\sup \left(j\left[\lambda_{i}\right]\right) .
$$

Notice that $g(i)<j\left(\lambda_{i}\right)$ since $j\left(\lambda_{i}\right)$ is regular in $V$, so $g$ is well defined. Notice also that $g$ dominates each $j\left(f_{\alpha}\right)$, since

$$
j\left(f_{\alpha}\right)(i)=j\left(f_{\alpha}\right)(j(i))=j\left(f_{\alpha}(i)\right)<g(i)
$$

for all $i$, so $j[\vec{f}]$ is not a scale. Contradiction.

CASE 2. $\lambda$ is regular.

Then $\lambda^{+\lambda}$ is a fixed point of $j$, singular, and limit of regular cardinals $\tau$ such that $j(\tau)>\tau$. We can then easily modify the argument of Case 1 so it applies to $\lambda^{+\lambda}$.

We have shown that for all $\lambda>\kappa, j(\lambda)>\lambda$. Now we prove the last assertion of the theorem. If $j[\lambda] \subseteq \lambda$ and $\operatorname{cf}^{M}(\lambda)<\kappa$, let $A \subseteq \lambda$ be in $M$ cofinal and of order type $\operatorname{cf}^{M}(\lambda)$. Then $j(A)=j[A] \subseteq \lambda$ and $j(\lambda)=\lambda$, a contradiction. This completes the proof.

It follows from this result that the critical point of a cardinal preserving embedding into $V$ is $\Pi_{1}$-indescribable in a very strong sense. For example:

COROLlary 2.9. If there is a cardinal preserving $j: M \rightarrow V$ then there is a proper class of weakly inaccessible cardinals.

Proof. Let $\kappa=\operatorname{cp}(j)$. Any weakly inaccessible cardinal $\lambda$ in $V$ is also weakly inaccessible in $M$ and therefore $j(\lambda)$ is (another) weakly inaccessible cardinal. $\kappa$ is weakly inaccessible in $M$, so there are (in $V$, thus in $M$ ) weakly inaccessible cardinals above $\kappa$. If there are only set many of them, their supremum would be a fixed point of $j$.

From Theorem 2.5 and Viale's Theorem 1.8, we immediately obtain:

Corollary 2.10. If $j: M \rightarrow V$ is cardinal preserving, PFA fails and any strongly compact cardinal is larger than $\mathrm{cp}(j)$.

Proof. That PFA fails follows from Theorem 1.8.7 (itself a consequence of items 4 and 6 of Theorem 1.8), since we can find (arbitrarily large) cardinals 
$\mu$ closed under $j$ and of $V$-cofinality $\omega$, but any such $\mu$ has $M$-cofinality at least $\mathrm{cp}(j)$, by Theorem 2.5; for example,

$$
\mu=j^{\omega}(\operatorname{cp}(j)):=\sup \{\operatorname{cp}(j), j(\operatorname{cp}(j)), j(j(\operatorname{cp}(j))), \ldots\} .
$$

If $\lambda<\operatorname{cp}(j)$ is strongly compact, then $\mathrm{CP}(\tau, \omega)$ holds for all regular cardinals $\tau \geq \lambda$, by Theorem 1.8.5. In particular, no $M$-regular cardinal above (or equal to) $\operatorname{cp}(j)$ can have $V$-cofinality $\omega$, by Theorem 1.8.6. But then we reach a contradiction exactly as above.

It also follows in the same way that if $j: M \rightarrow V$ is cardinal preserving and $\tau$ is strongly compact, then for any $\lambda \geq \tau, \operatorname{cf}^{M}(\lambda) \geq \tau$ iff $\operatorname{cf}(\lambda) \geq \tau$.

2.2. Consistency strength. Let $j: M \rightarrow V$ be cardinal preserving. Since such an $M$ computes incorrectly many cofinalities, covering fails very badly for $M$, and it should be no surprise that such an embedding would require considerable consistency strength. For example:

THEOREM 2.11. Assume that there is a cardinal preserving embedding $j$ : $M \rightarrow V$. Then there are inner models with strong cardinals.

Proof. Assume otherwise. Then $K$ exists, is rigid, and satisfies the weak covering property. In particular, $K$ computes cofinally many successor cardinals correctly.

Universality of a class $W=L[\mathcal{E}]$ is a technical assumption, see Steel [19] for a definition, but below strong cardinals, it follows by the covering lemma, see Mitchell [15], that if $\left(\mu^{+}\right)^{W}=\mu^{+}$for cofinally many cardinals $\mu$, then $W$ is universal. By Mitchell [15], any universal proper class mouse $W$ is an iterate of $K$, so in particular there is an elementary $\pi: K \rightarrow W$.

Since we are below strong cardinals, $K^{M}$ is iterable in $V$ (since, below Woodin cardinals, iterability can be expressed as a $\Pi_{2}^{1}$ condition) and it follows that it is universal by the weak covering property (in $M$ ) and the fact that $\mathrm{CAR}^{M}=\mathrm{CAR}$. But then there is an embedding $\pi: K \rightarrow K^{M}$, so $j \circ \pi$ : $K \rightarrow K$ is nontrivial. Contradiction.

§3. Embeddings of $V$. Now we turn our attention to the case of embeddings $j: V \rightarrow M$.

3.1. Nice cardinals. We start by showing that cardinal preserving embeddings must have significant large cardinal strength.

Definition 3.1. Given a cardinal preserving $j: V \rightarrow M$, we say that a regular cardinal $\mu$ is $j$-nice (or nice, if $j$ is clear from context) iff

$$
\delta_{\mu}:=\sup j[\mu]<j(\mu) .
$$

Throughout this section (even if not explicitly stated), $j: V \rightarrow M$ is cardinal preserving and $\kappa=\mathrm{cp}(j)$. If $\mu$ is $j$-nice, then we can define a uniform 
$\kappa$-complete ultrafilter $\mathcal{U}_{\mu}$ on $\mu$ by setting

$$
\mathcal{U}_{\mu}:=\left\{X \subseteq \mu: \delta_{\mu} \in j(X)\right\} .
$$

The point is that there are many $j$-nice cardinals $\mu$. For example, $\mu$ is $j$-nice if $\mu=\rho^{+}$and $\rho<j(\rho)$, because sup $j\left[\rho^{+}\right]$has cofinality $\rho^{+}$while $j\left(\rho^{+}\right)=$ $j(\rho)^{+}$is regular and larger than $\rho^{+}$since $j(\rho)>\rho$.

THEOREM 3.2. If there is a cardinal preserving $j: V \rightarrow M$, then $V$ is closed under sharps.

Proof. Let $A$ be a set, which we may assume transitive. If $A^{\sharp}$ does not exist, by the covering lemma, see Mitchell [15], whenever $\lambda$ is a singular strong limit cardinal larger than $\operatorname{rk}(A)$, then $\lambda^{+}=\left(\lambda^{+}\right)^{L(A)}$. Fix such $\lambda$ of cofinality $\kappa$. Then $\lambda^{+}$is nice. As in the Vopěnka-Hrbáček argument from strongly compact cardinals, see Kanamori [10, Theorem 5.9], we can then consider the two embeddings $j_{\lambda^{+}}: V \rightarrow M_{\lambda^{+}} \cong \operatorname{ult}\left(V, \mathcal{U}_{\lambda^{+}}\right)$and $k: V \rightarrow N_{\lambda^{+}} \cong \operatorname{ult}^{-}\left(V, \mathcal{U}_{\lambda^{+}}\right)$, where ult ${ }^{-}$is formed by only considering those functions $f: \lambda^{+} \rightarrow V$ such that $|\operatorname{ran}(f)|<\lambda^{+}$. We then also have an embedding $i: N_{\lambda^{+}} \rightarrow M_{\lambda^{+}}$given by $i\left([f]^{-}\right)=[f]$, where $[f]^{-}$is the collapse of the equivalence class of $f$ in the ultrapower $\mathrm{ult}^{-}\left(V, \mathcal{U}_{\lambda^{+}}\right)$and $[f]$ is the collapse of the corresponding class in $\operatorname{ult}\left(V, \mathcal{U}_{\lambda^{+}}\right)$. We then have (see Kanamori [10, Theorem 5.9]):

- $j_{\lambda^{+}}=i \circ k$.

- $\operatorname{cp}(i) \geq k\left(\lambda^{+}\right)$and $j_{\lambda^{+}}(A)=k(A)$.

- $k\left(\lambda^{+}\right)<j_{\lambda^{+}}\left(\lambda^{+}\right)$.

But then it follows that $j_{\lambda^{+}}(\lambda)=k(\lambda)$ and

$$
\begin{aligned}
j_{\lambda^{+}}\left(\lambda^{+}\right) & =j_{\lambda^{+}}\left(\left(\lambda^{+}\right)^{L(A)}\right)=\left(j_{\lambda^{+}}(\lambda)^{+}\right)^{L\left(j_{\lambda^{+}}(A)\right)} \\
& =\left(k(\lambda)^{+}\right)^{L(k(A))}=k\left(\left(\lambda^{+}\right)^{L(A)}\right) \\
& =k\left(\lambda^{+}\right),
\end{aligned}
$$

contradiction.

Schindler pointed out that essentially the same argument gives more information:

THEOREM 3.3. If there is a cardinal preserving $j: V \rightarrow M$, then for all $n$ and all $X, M_{n}^{\sharp}(X)$ exists.

We omit the (technical) proof of Theorem 3.3, but include a (very brief) sketch for the experts: The result follows by induction on $n$. Assuming, for example, that $M_{n}^{\sharp}(X)$ exists for all $X$ but $M_{n+1}^{\sharp}$ does not, if one lets $E$ be a long extender coding a sufficiently large fragment of $j$, then one can build $K$ inside $M_{n}^{\sharp}(E)$. In $M_{n}^{\sharp}(E)$ there is then an elementary embedding (coming from $E$, as above) sending $K$ to a universal weasel such that the map is discontinuous at some successor cardinal where covering holds (just as before). This gives a contradiction, exactly as above. 
The cardinal $\kappa=\mathrm{cp}(j)$ possesses very strong large cardinal properties just shy of strong compactness. For example, cofinally often, $\mu$ is nice and therefore the ultrafilter $\mathcal{U}_{\mu}$ is defined; in particular, this holds for all regular cardinals $\mu \geq \kappa$ below $\lambda_{j}$, where

$$
\lambda_{j}=\min \{\text { first weakly inaccessible above } \kappa \text {, first fixed point of } j \text { above } \kappa\} \text {, }
$$

since all these cardinals $\mu$ are of the form $\rho^{+}$for some $\rho$ such that $\rho<j(\rho)$. Ketonen [12] has shown that an uncountable regular cardinal $v$ is $\tau$-compact for regular $\tau \geq v$ iff all regular $\mu$ with $v \leq \mu \leq \tau$ carry a uniform $v$-complete ultrafilter. Thus:

COROLLARY 3.4. If there is a cardinal preserving $j: V \rightarrow M$ with $\kappa=\operatorname{cp}(j)$, and $\lambda_{j}$ is defined as above, then $\kappa$ is $<\lambda_{j}$-strongly compact.

In particular, $\kappa$ is $\kappa^{+}$-strongly compact, so $\kappa$ is a measurable cardinal such that $\square_{\kappa}$ fails, see Kanamori-Magidor [11]. It follows from Andretta-NeemanSteel [1] that there is a non-domestic premouse and in particular there are inner models of $Z F+A D_{\mathbb{R}}$ containing all the reals.

The following is immediate from the proof of Viale's Theorem 1.8, see Viale [23]:

THEOREM 3.5. Given a cardinal preserving $j: V \rightarrow M$, if $\lambda$ is $j$-nice, then the covering property $\operatorname{CP}(\lambda, \theta)$ holds for all regular $\theta<\kappa=\operatorname{cp}(j)$ and therefore

1. $\square(\lambda)$ fails.

2. If $\lambda=\rho^{+}$and $\operatorname{cf}(\rho)=\theta<\kappa$, then $\lambda^{\theta}=\lambda$.

In particular, if $\lambda=\rho^{+}$is nice, then either $\rho$ is singular in $M$ or else $\operatorname{cf}(\rho) \geq \kappa$.

THEOREM 3.6. If $j: V \rightarrow M$ is cardinal preserving, then $j$ is not the ultrapower embedding by a set extender.

Proof. We proceed as before: Let $E$ be a $(\kappa, \beta)$-extender with each $E_{a}$ over $[\zeta]^{|a|}$, and let $j: V \rightarrow M$ be the corresponding embedding. Towards a contradiction, suppose that $j$ is cardinal preserving. Let $\lambda>\zeta, \beta$ be a singular strong limit cardinal of cofinality $\kappa$ so, in particular, $\lambda>2^{\kappa}$. Then $\lambda<j(\lambda)$ and since $j(\lambda)$ is a singular cardinal of $M$-cofinality $j(\kappa), j(\lambda) \geq \lambda^{+\kappa}$. Since $2^{\lambda}=\lambda^{\kappa}=j\left(\lambda^{\kappa}\right)>j(\lambda), \lambda$ violates the singular cardinal hypothesis $\mathrm{SCH}$; here, $\lambda^{\kappa}=j\left(\lambda^{\kappa}\right)$ follows as before: $j\left(\lambda^{\kappa}\right)<\left(\lambda^{\kappa|\xi|}|\beta|\right)^{+}$, by Corollary 1.3. It follows that $\lambda^{+\kappa}$ also violates $\mathrm{SCH}$, since $\lambda^{+\kappa}<\left(\lambda^{+\kappa}\right)^{\kappa}=\lambda^{\kappa}$ and, since $\lambda^{\kappa}$ is fixed by $j$, then

$$
\lambda^{+\kappa+1}<j(\lambda)^{+j(\kappa)}=j\left(\lambda^{+\kappa}\right)<j\left(\lambda^{\kappa}\right)=\lambda^{\kappa} .
$$

By Silver's theorem or the Galvin-Hajnal results, see for example HolzSteffens-Weitz [7, Corollary 2.3.4], $\left\{\mu<\lambda^{+\kappa}: \mu^{\omega}>\mu^{+}\right\}$contains an $\omega$-club. For $\mu$ singular of cofinality $\omega$, recall that $\tau=\mu^{+}$is nice if $j(\mu)>\mu$, and that therefore $\mathrm{CP}(\tau, \omega)$ holds, so $\tau^{\omega}=\tau$, or $\mu^{\omega}=\mu^{+}$. Hence, $j(\mu)=\mu$ 
for an $\omega$-club of cardinals below $\lambda^{+\kappa}$. However, all cardinals in the interval $\left[\lambda, \lambda^{+\kappa}\right)$ are moved by $j$. Contradiction.

3.2. Cofinality preserving embeddings. We close the paper by showing how consistency strength can be extracted in a cleaner way from an embedding $j: V \rightarrow M$ if we impose the formally stronger requirement that $M$ computes cofinalities correctly.

Theorem 3.7 (Caicedo, Woodin). Assume $j: V \rightarrow M$ is such that if $\kappa=$ $\operatorname{cp}(j)$, then $\operatorname{cf}(\lambda)=\operatorname{cf}^{M}(\lambda)$ for any $\lambda \leq \sup j[j(\kappa)]$. Then

1. $j(\kappa)$ is strongly inaccessible and

2. $V_{j(\kappa)}=\kappa$ is strongly compact.

Proof. The assumption guarantees that $\lambda$ is nice whenever $\kappa \leq \lambda \leq j(\kappa)$ and $\lambda$ is regular, and therefore $\kappa$ is $\leq j(\kappa)$-strongly compact. We start by showing that $j$ witnesses the $<j(\kappa)$-strong compactness of $\kappa$ in the sense that for any $\lambda<j(\kappa)$ there is in $M$ a set $Y$ such that $j[\lambda] \subset Y$ and $|Y|<j(\kappa)$. For this, fix in $V$ a sequence

$$
C=\left(C_{\alpha}: \alpha<\lambda\right)
$$

such that each $C_{\alpha}$ is a club subset of $\alpha$ of order type $\operatorname{cf}(\alpha)$. Then $j(C)_{\delta_{\lambda}}$ is club in $\delta_{\lambda}$ and has order type $\lambda$. Let $D=\left\{\beta<\lambda: j(\beta) \in j(C)_{\delta_{\lambda}}\right\}$. Then $D$ is $<\kappa$-club in $\lambda$ and $j[D] \subseteq j(C)_{\delta_{\lambda}}$.

Now use a bijection $\pi: D \rightarrow \lambda$ to lift the covering of $j[D]$ to a covering of $j[\lambda]$ : Notice that $j(\pi)$ is a bijection between $j(D)$ and $j(\lambda)$, and that $j(\pi)[j[D]]=j[\lambda]$.

The same argument shows that $j[j(\kappa)]$ is covered by a set in $M$ of size $j(\kappa)$.

We use this covering property of $j$ to establish the strong inaccessibility of $j(\kappa)$. For suppose that there is a regular $\gamma, \kappa \leq \gamma<j(\kappa)$, such that $2^{\gamma} \geq j(\kappa)$. Fix $X \subseteq \mathcal{P}(\gamma)$ of size $j(\kappa)$. Then there is $Y \in M$ of size $j(\kappa)$ covering $j[X]$. Clearly $a \cap j[\gamma] \neq b \cap j[\gamma]$ whenever $a \neq b \in j[X]$. Let $S \in M$ cover $j[\gamma]$ and have size $<j(\kappa)$. It follows that $Y \cap \mathcal{P}(S)$ has size $j(\kappa)$ in $M$ and therefore $j(\kappa)$ is not strongly inaccessible in $M$. Contradiction.

Finally, since any ultrafilter on $\mathcal{P}_{\kappa}(\lambda), \lambda<j(\kappa)$, lives in $V_{j(\kappa)}$, clearly $V_{j(\kappa)}=\mathrm{ZFC}+\kappa$ is strongly compact.

Notice that the assumptions of Theorem 3.7 are not (expected to be) vacuous, since for example a 2-huge cardinal induces an embedding as required in Theorem 3.7. What is interesting is that we have recovered a very strong hypothesis from an assumption that rather than directly imposing closure on the target model only requires of it some degree of "correctness." Recall that $\kappa$ is 2-huge iff there is an embedding $j: V \rightarrow M$ with $\mathrm{cp}(j)=\kappa$ and such that $M^{j(j(\kappa))} \subseteq M$. In fact, the weaker assumption of a 2-superstrong cardinal suffices. Recall that $\kappa$ is $n$-superstrong iff there is an embedding $j: V \rightarrow M$ with $\operatorname{cp}(j)=\kappa$ and $V_{j^{n}(\kappa)} \subseteq M$, where the superscript indicates iteration. These are in any case significant assumptions; while superstrong cardinals are 
"weak" in the sense that any supercompact cardinal is limit of superstrong cardinals, 2-superstrength is (consistency-wise) above supercompactness.

Question 3.8. Is it consistent to have an embedding $j: V \rightarrow M$ such that the first $\lambda^{+\kappa+1}$ cardinals of $M$ and of $V$ coincide? Here, $\kappa=\operatorname{cp}(j)$ and $\lambda$ is the first fixed point of $j$ above $\kappa$.

Acknowledgments. Some results in this paper strengthen results first announced in the invited talk Cardinal preserving elementary embeddings given in Oaxaca, México, on August 2006 during the XIII SLALM; I want to thank the organizing committee of the SLALM for allowing me the opportunity to speak, and the NSF for support in attending the meeting through grant DMS-0605727. The new results were to form part of the talk I was to present during the Logic Colloquium 2007, but health issues prevented me from attending; I want to thank the ASL and the organizing committee of the Colloquium for the invitation and the opportunity to include this paper in its proceedings. I also want to thank Grigor Sargsyan for conversations regarding Section 3, and the referees for their useful suggestions.

\section{REFERENCES}

[1] A. Andretta, I. Neeman, and J. Steel, The domestic levels of $K^{c}$ are iterable, Israel Journal of Mathematics, vol. 125 (2001), pp. 157-201.

[2] J. Baumgartner, Applications of the proper forcing axiom, Handbook of Set-Theoretic Topology (K. Kunen and J. Vaughan, editors), North-Holland, Amsterdam, 1984, pp. 913-959.

[3] M. BekKali, Topics in Set Theory, Lecture Notes in Mathematics, vol. 1476, SpringerVerlag, Berlin, 1991, Lebesgue measurability, large cardinals, forcing axioms, rho-functions, Notes on lectures by Stevo Todorčević.

[4] A. Caicedo and B. VeličKović, Properness and Reflection Principles in Set Theory, in preparation.

[5] M. Foreman, M. Magidor, and S. Shelah, Martin's maximum, saturated ideals, and nonregular ultrafilters. I, Annals of Mathematics. Second Series, vol. 127 (1988), no. 1, pp. 1-47.

[6] J. Hamkins, Forcing and Large Cardinals, in preparation.

[7] M. Holz, K. Steffens, and E. Weitz, Introduction to Cardinal Arithmetic, Birkhäuser Advanced Texts: Basler Lehrbücher. [Birkhäuser Advanced Texts: Basel Textbooks], Birkhäuser Verlag, Basel, 1999.

[8] T. JeCh, Set Theory, Springer Monographs in Mathematics, Springer-Verlag, Berlin, 2003, The third millennium edition, revised and expanded.

[9] R. Jensen, E. Schimmerling, R. Schindler, and J. Steel, Stacking mice, The Journal of Symbolic Logic, vol. 74 (2009), no. 1, pp. 315-335.

[10] A. Kanamori, The Higher Infinite: Large Cardinals in Set Theory from Their Beginnings, second ed., Springer Monographs in Mathematics, Springer-Verlag, Berlin, 2003.

[11] A. Kanamori and M. Magidor, The evolution of large cardinal axioms in set theory, Higher Set Theory (Proc. Conf., Math. Forschungsinst., Oberwolfach, 1977), Lecture Notes in Mathematics, vol. 669, Springer, Berlin, 1978, pp. 99-275.

[12] J. Ketonen, Strong compactness and other cardinal sins, Annals of Pure and Applied Logic, vol. $5(1972 / 73)$, pp. $47-76$. 
[13] P. LaRson, The Stationary Tower: Notes on a Course by W. Hugh Woodin, University Lecture Series, vol. 32, American Mathematical Society, Providence, RI, 2004.

[14] D. A. Martin and R. M. Solovay, Internal Cohen extensions, Annals of Pure and Applied Logic, vol. 2 (1970), no. 2, pp. 143-178.

[15] W. Mitchell, The Covering Lemma, to appear in Handbook of Set Theory, Foreman and Kanamori (Editors).

[16] J. Moore, Set mapping reflection, Journal of Mathematical Logic, vol. 5 (2005), no. 1, pp. 87-97.

[17] S. Shelah, Cardinal Arithmetic, Oxford Logic Guides, vol. 29, The Clarendon Press Oxford University Press, New York, 1994.

[18] _ Proper and Improper Forcing, second ed., Perspectives in Mathematical Logic, Springer-Verlag, Berlin, 1998.

[19] J. Steel, An Outline of Inner Model Theory, to appear in Handbook of Set Theory, Foreman and Kanamori (Editors).

[20] S. TodorČEvić, A note on the proper forcing axiom, Axiomatic Set Theory (Boulder, Colorado, 1983) (J. Baumgartner and D. Martin, editors), Contemporary Mathematics, vol. 31, American Mathematical Society, Providence, RI, 1984, pp. 209-218.

[21] M. VIALE, Applications of the proper forcing axiom to cardinal arithmetic, Ph.D. Dissertation, Université Paris 7, Denis Diderot, Paris, 2006.

[22] - The proper forcing axiom and the singular cardinal hypothesis, The Journal of Symbolic Logic, vol. 71 (2006), no. 2, pp. 473-479.

[23] - A family of covering properties, Mathematical Research Letters, vol. 15 (2008), no. 2, pp. 221-238.

[24] J. VICKERS and P. D. WELCH, On elementary embeddings from an inner model to the universe, The Journal of Symbolic Logic, vol. 66 (2001), no. 3, pp. 1090-1116.

[25] J. Zapletal, A new proof of Kunen's inconsistency, Proceedings of the American Mathematical Society, vol. 124 (1996), no. 7, pp. 2203-2204.

\section{CALIFORNIA INSTITUTE OF TECHNOLOGY}

DEPARTMENT OF MATHEMATICS

MAIL CODE 253-37

PASADENA, CA 91125 , USA

Current address: Department of Mathematics, Boise State University, 1910 University Drive, Boise, ID 83725, USA

E-mail: caicedo@math.boisestate.edu

URL: http://math.boisestate.edu/ caicedo/ 